\title{
Astrocyte activation in the anterior cingulate cortex and altered glutamatergic gene expression during paclitaxel- induced neuropathic pain in mice
}

Willias Masocha

Spinal astrocyte activation contributes to the pathogenesis of paclitaxel-induced neuropathic pain (PINP) in animal models. We examined glial fibrillary acidic protein (GFAP; an astrocyte marker) immunoreactivity and gene expression of GFAP, glutamate transporters and receptor subunits by real time PCR in the anterior cingulate cortex (ACC) at 7 days post first administration of paclitaxel, a time point when mice had developed thermal hyperalgesia. The ACC, an area in the brain involved in pain perception and modulation, was chosen because changes in this area might contribute to the pathophysiology of PINP. GFAP transcripts levels were elevated by more than fivefold and GFAP immunoreactivity increased in the ACC of paclitaxel-treated mice. The 6 glutamate transporters (GLAST, GLT-1 EAAC1, EAAT4, VGLUT-1 and VGLUT-2) quantified were not significantly altered by paclitaxel treatment. Of the 12 ionotropic glutamate receptor subunits transcripts analysed 6 (GLuA1, GLuA3, GLuK2, GLuK3, GLuK5 and GLuN1) were significantly up-regulated, whereas GLuA2, GLuK1, GLuK4, GLuN2A and GLuN2B were not significantly altered and GLuA4 was lowly expressed. Amongst the 8 metabotropic receptor subunits analysed only mGLuR8 was significantly elevated. In conclusion, during PINP there is astrocyte activation, no change in glutamate transporter expression and differential up-regulation of glutamate receptor subunits in the ACC. Thus, targeting astrocyte activation and the glutamatergic system might be another therapeutic avenue for management of PINP. 
1 Astrocyte activation in the anterior cingulate cortex and altered glutamatergic gene

2 expression during paclitaxel-induced neuropathic pain in mice

3 Abstract

4 Spinal astrocyte activation contributes to the pathogenesis of paclitaxel-induced neuropathic pain

5 (PINP) in animal models. We examined glial fibrillary acidic protein (GFAP; an astrocyte

6 marker) immunoreactivity and gene expression of GFAP, glutamate transporters and receptor

7 subunits by real time PCR in the anterior cingulate cortex (ACC) at 7 days post first

8 administration of paclitaxel, a time point when mice had developed thermal hyperalgesia. The

9 ACC, an area in the brain involved in pain perception and modulation, was chosen because

10 changes in this area might contribute to the pathophysiology of PINP. GFAP transcripts levels

11 were elevated by more than fivefold and GFAP immunoreactivity increased in the ACC of

12 paclitaxel-treated mice. The 6 glutamate transporters (GLAST, GLT-1 EAAC1, EAAT4,

13 VGLUT-1 and VGLUT-2) quantified were not significantly altered by paclitaxel treatment. Of

14 the 12 ionotropic glutamate receptor subunits transcripts analysed 6 (GLuA1, GLuA3, GLuK2,

15 GLuK3, GLuK5 and GLuN1) were significantly up-regulated, whereas GLuA2, GLuK1,

16 GLuK4, GLuN2A and GLuN2B were not significantly altered and GLuA4 was lowly expressed.

17 Amongst the 8 metabotropic receptor subunits analysed only $\mathrm{mGLuR}_{8}$ was significantly

18 elevated. In conclusion, during PINP there is astrocyte activation, no change in glutamate

19 transporter expression and differential up-regulation of glutamate receptor subunits in the ACC.

20 Thus, targeting astrocyte activation and the glutamatergic system might be another therapeutic

21 avenue for management of PINP.

22 Willias Masocha 
23 Department Pharmacology and Therapeutics, Faculty of Pharmacy, Kuwait University, Safat,

24 Kuwait

25 Phone number: +965 24636078 Email: masocha@hsc.edu.kw

26 Introduction

27 The anterior cingulate cortex (ACC) is a cortical area in the brain that has been described to be involved with pain possibly including both perception and modulation (Vogt 2005; Xie et al. 2009; Zhuo 2008). It is a component of the medial pain pathway. The afferent inputs to the ACC are from midline and intralaminar thalamic nuclei, whilst the ACC sends projections into various areas including the intralaminar thalamic nuclei and periaqueductal grey (PAG, which is involved in control of descending pain) (Senapati et al. 2005; Sewards \& Sewards 2002; Vogt 2005). Neuroimaging studies have shown increased activity in the ACC during chronic pain, including neuropathic pain (Hsieh et al. 1995; Peyron et al. 2000; Tseng et al. 2013). Neurophysiological and molecular changes have also been observed in the ACC during chronic or neuropathic (Wrigley et al. 2009; Xu et al. 2008; Yamashita et al. 2014).

One of the changes that has been observed in the ACC during chronic or neuropathic pain is the activation of astrocytes or astrogliosis (Chen et al. 2012; Kuzumaki et al. 2007; Lu et al. 2011; Narita et al. 2006; Yamashita et al. 2014). Astrocytes are the most numerous non-neuronal cells in the brain involved in modulation of neuronal activities e.g. extracellular and synaptic cleft neurotransmitter level regulation, release of neuroactive molecules amongst other activities (Maragakis \& Rothstein 2006; Seifert et al. 2006). Astrocytes express transporters which remove neurotransmitters such as $\gamma$-aminobutyric acid (GABA) and glutamate from the extracellular space or synaptic cleft (Conti et al. 1998; Danbolt 2001; Gosselin et al. 2010; Minelli et al. 1995; 
46

47

48

49

50

51

52

GABA and a decrease in transporters for glutamate resulting in a more excitatory state in the brain (Gosselin et al. 2010; Maragakis \& Rothstein 2006). Recently, we observed an increase in the transcripts of GABA transporter 1 (GAT-1) in a rodent model of paclitaxel-induced neuropathic pain (PINP) (Masocha 2015). However, it is not known whether paclitaxel induces astrocyte activation in the ACC although it has been shown to induce astrocyte activation in the spinal cord (Peters et al. 2007; Zhang et al. 2012). Paclitaxel is a chemotherapeutic agent that causes dose-dependent neuropathic pain in some patients (Scripture et al. 2006; Wolf et al. 2008). In the rodent models we observed that the PINP is linked with disturbances in the GABAergic system (Masocha 2015) resulting in increased excitability of the ACC to electrophysiological stimulation (H Nashawi, IO Edafiogho, SB Kombian, W Masocha, unpublished data). GABA is the major inhibitory neurotransmitter while glutamate is the major stimulatory neurotransmitter in the brain (Meldrum 2000; Petroff 2002). It is not known whether paclitaxel causes any changes in the glutamatergic system in the ACC, although it has been shown to decrease the expression of glutamate transporters such as GLAST and GLT-1 in the spinal cord (Weng et al. 2005; Zhang et al. 2012). There are 8 known glutamate transporters, which are excitatory amino acid transporter 1 (EAAT1; referred to as GLAST in rodents), EAAT2 (GLT-1), EAAT3 (EAAC1), EAAT4, EAAT5, vesicular glutamate transporter 1 (VGLUT1), VGLUT2, and VGLUT3 (Danbolt 2001; Shigeri et al. 2004). Of the transporters GLAST and GLT-1 are expressed on astrocytes (Danbolt 2001) and play an important role in removal of glutamate from the synaptic cleft and extracellular space (Danbolt 2001; Shigeri et al. 2004) and if their expression is down-regulated this results in increased levels of glutamate and excitotoxicity (Danbolt 2001; Rothstein et al. 1996; Shigeri et al. 2004; Yi et al. 2005). Glutamate acts on ionotropic and metabotropic receptors. The ionotropic receptors are divided 
69 into alpha-amino-3-hydroxy-5-methyl-4-isoxazolpropionate (AMPA), kainate and N-methyl-D-

70 aspartate (NMDA) receptors which have 18 subunits GLuA1 to 4, GLuK1-5 and GLuN1,

71 GLuN2A to D, GLuN3A and B, and GLuD1 and 2 (Collingridge et al. 2009). There are 8

72 subunits of the metabotropic receptors mGLUR $_{1}$ to ${ }_{8}$ (Conn \& Pin 1997; Niswender \& Conn

73 2010).

74 Astrocyte activation, which has been observed in the ACC in models of chronic and neuropathic

75 pain (Chen et al. 2012; Kuzumaki et al. 2007; Lu et al. 2011; Narita et al. 2006; Yamashita et al.

76 2014), might occur in the ACC during PINP together with molecular changes in the

77 glutamatergic system contributing to the pathogenesis or maintenance of PINP. Thus, in this

78 study, astrocyte activation and the gene expression of molecules of the astrocyte marker (glial

79 fibrillary acidic protein (GFAP), glutamate transporters and receptors in the ACC were evaluated

80 in mice at a time point when the mice had paclitaxel-induced thermal hyperalgesia (Nieto et al.

81 2008; Parvathy \& Masocha 2013). 


\section{Materials and Methods}

\section{Animals}

84 Ninety eight female BALB/c mice ( 8 to 12 weeks old) supplied by the Animal Resources Centre 85 (ARC) at the Health Sciences Center (HSC), Kuwait University were used. The animals were 86 housed and handled in compliance with the Kuwait University, HSC, ARC guidelines and 87 published ethical guidelines for research in experimental pain with conscious animals 88 (Zimmermann 1983). All animal experiments were approved by the Ethical Committee for the 89 use of Laboratory Animals in Teaching and in Research, HSC, Kuwait University.

\section{Paclitaxel administration}

Paclitaxel (Cat. No. 1097, Tocris, Bristol, UK) was dissolved in a solution made up of 50\% Cremophor EL and 50\% absolute ethanol to a concentration of $6 \mathrm{mg} / \mathrm{ml}$ and then diluted in normal saline $(\mathrm{NaCl} 0.9 \%)$, to a final concentration of $0.2 \mathrm{mg} / \mathrm{ml}$ just before administration. The vehicle for paclitaxel, thus, constituted of about $1.7 \%$ Cremophor EL and $1.7 \%$ ethanol in normal saline. Paclitaxel $2 \mathrm{mg} / \mathrm{kg}$ or its vehicle were administered to mice intraperitoneally (i.p.), daily for 5 consecutive days. This treatment regimen has been reported to produce painful neuropathy and thermal hyperalgesia in mice (Nieto et al. 2008; Parvathy \& Masocha 2013).

\section{Hot plate test}

Reaction latencies to hot plate test were measured before (baseline latency) and on day 7 after first administration of paclitaxel. Briefly, mice were placed on a hot plate (Panlab SL, Barcelona, Spain) with the temperature adjusted to $55 \pm 1^{\circ} \mathrm{C}$. The time to the first sign of nociception, paw 
102 licking or flinching, was recorded and the animal immediately removed from the hot plate. A

103 cut-off period of 20 seconds was maintained to avoid damage to the paws.

\section{ACC tissue preparation}

105 The mice were anesthetized with isoflurane and sacrificed by decapitation. ACC was dissected and prepared for RNA extraction on day 7 post first administration of paclitaxel — a time point when mice had developed thermal hyperalgesia (Parvathy \& Masocha 2013) —, as described previously (Masocha 2015)

\section{Real time RT-PCR}

Gene transcripts of the astrocyte marker GFAP, 6 glutamate transporters (GLAST, GLT-1,

111 EAAC1, EAAT4, VGLUT1, VGLUT2), 12 ionotropic glutamate receptor subunits (GLuA1 to 4,

112 GLuK1 to 5, GLuN1, GLuN2A and GLuN2B) and 8 metabotropic glutamate subunits (mGluR 1

113 to ${ }_{8}$ ) were quantified in the ACC of vehicle-treated or paclitaxel-treated by real time PCR. Total

114 RNA was extracted from the fresh frozen ACC using the RNeasy Kit (Qiagen GmbH), reverse-

115 transcribed, and the mRNA levels were quantified on an ABI Prism ${ }^{\circledR} 7500$ sequence detection

116 system (Applied Biosystems) as previously described (Masocha 2009). The primer sequences

117 which were used, listed in Table 1, were ordered from Invitrogen (Life Technologies) and/or

118 synthesized at the Research Core Facility (RCF), HSC, Kuwait University. The amplification

119 and detection were performed as follows: a first hold at $50^{\circ} \mathrm{C}$ for 2 min, a second hold at $95^{\circ} \mathrm{C}$

120 for $2 \mathrm{~min}$ followed by 40 cycles at $95^{\circ} \mathrm{C}$ for $15 \mathrm{~s}$ and $63^{\circ} \mathrm{C}$ for $1 \mathrm{~min}$. Threshold cycle $(\mathrm{Ct})$

121 values for all cDNA samples were obtained and the amount of mRNA of individual animal

122 sample ( $\mathrm{n}=6$ to 24 per group) was normalized to cyclophilin (housekeeping gene) $(\Delta \mathrm{Ct})$. The 
123 relative amount of target gene transcripts was calculated using the $2^{-\Delta \Delta C t}$ method as described

124 previously (Livak \& Schmittgen 2001).

\section{Immunohistochemistry}

126 Fresh-frozen brains were cut on a cryostat into $25 \mu \mathrm{m}$ thick sections and thaw-mounted on

127 chrome-alum gelatin-coated slides. The sections at a level of the lateral ventricles and the ACC

128 were fixed in $4 \%$ formalin and $14 \%$ picric acid in $\mathrm{PBS}$ for $30 \mathrm{~s}$ at $4^{\circ} \mathrm{C}$, rinsed in $\mathrm{PBS}$, fixed in

129 acetone for $30 \mathrm{~s}$ at $-20^{\circ} \mathrm{C}$, and then rinsed in PBS. All sections were preincubated with $1 \%$

130 bovine serum albumin and $0.3 \%$ Triton X-100 in PBS (solution used as diluent for primary and

131 secondary antibodies) for $30 \mathrm{~min}$ at room temperature. Sections were incubated with rabbit anti-

132 GFAP (1:100; DAKO, Glostrup, Denmark) for $2 \mathrm{~h}$ at room temperature to immunostain

133 astrocytes. Sections were then rinsed in PBS and incubated with DyLight 594-conjugated

134 Affinipure donkey Anti-rabbit IgG $(\mathrm{H}+\mathrm{L})(1: 100$, Jackson ImmunoResearch Laboratories, West

135 Grove, PA, USA) for $1 \mathrm{~h}$. The sections were rinsed in PBS and mounted in ProLong® Gold

136 antifade reagent (Invitrogen, USA). Sections were examined and analysed using a LSM 700 laser

137 scanning confocal microscope. Images were taken from the ACC using an Axio imager (Carl

138 Zeiss MicroImaging GmbH, Germany).

\section{Statistical analyses}

141 Statistical analyses were performed using unpaired two-tailed Student's t-test using Graph Pad

142 Prism software (version 5.0). The differences were considered significant at $p<0.05$. The results

143 in the text and figures are expressed as the means \pm S.E.M. 


\section{Results}

\section{Paclitaxel-induced thermal hyperalgesia}

146 Mice developed thermal hyperalgesia on day 7 after first administration of paclitaxel as we

147 previously described (Masocha 2014; Parvathy \& Masocha 2013) i.e. paclitaxel-treated mice had

148 significant reduction in response latency time in the hot plate test on day 7 compared to the

149 baseline latency and vehicle-treated animals $(6.23 \pm 0.28 \mathrm{~s}$ compared to $9.66 \pm 0.16 \mathrm{~s}$ and $9.00 \pm$

$1500.38 \mathrm{~s}$, respectively; $\mathrm{n}=10$ vehicle-treated mice and 16 paclitaxel treated-mice; $\mathrm{p}<0.05$ for both 151 comparisons).

Astrocyte activation in the $\mathrm{ACC}$ at 7 days after paclitaxel administration

The mRNA expression and immunoreactivity of the astrocyte marker, GFAP, were analysed in the ACC at day 7, a time when the mice had developed thermal hyperalgesia. Treatment with paclitaxel significantly increased the expression of GFAP transcripts $(p=0.02)$ by more than fivefold compared to vehicle-treated controls (Fig. 1). Confocal microscopy images showed that in paclitaxel-treated mice there was increased GFAP immunoreactivity in the ACC compared to vehicle-treated controls (Fig. 2). However, the change in GFAP immunoreactivity in paclitaxeltreated animals varied across the ACC and animals i.e. it was not robust in all animals and did not cover most of the ACC. 
164 Expression of transcripts of glutamate transporters in the ACC at 7 days after paclitaxel 165 administration

166 .There were no differences observed in the transcript levels of all the six glutamate transporters

167 analysed (Fig. 3) in the ACC of paclitaxel-treated mice compared to vehicle-treated mice. Using

168 the unpaired two-tailed Student's t-test the $\mathrm{p}$ values obtained are: 0.7243 for GLAST, 0.6608 for

169 GLT-1, 0.7575 for EAAC1, 0.5925 for EAAT4, 0.8885 for VGLUT-1 and 0.0858 for VGLUT$170 \quad 2 .$.

171

172

Expression of transcripts of glutamate receptors in the ACC at 7 days after paclitaxel

173

174

175

176

177

178

179

180

181

182

183

\section{administration}

Amongst the AMPA receptor subunits GLuA4 was lowly expressed in the ACC and mRNA expression was not detected after 40 cycles in the real time RT-PCR in 12 out of 16 vehicle- and paclitaxel-treated animals analysed. Treatment with paclitaxel did not significantly alter the mRNA expression of the AMPA receptor subunit GLuA2 $(p=0.9720)$, but significantly increased the expression of GLuA1 $(p=0.0166)$ and GLuA3 $(p=0.0243)$ subunits compared to vehicle-treated controls (Fig. 4A).

Amongst the 5 kainate receptor subunits analysed treatment with paclitaxel significantly increased the expression of the 3 subunits GluK2 $(p=0.0136)$, GluK3 $(p=0.0026)$ and GluK5 $(\mathrm{p}=0.0011)$, but not 2 subunits GluK1 $(\mathrm{p}=0.4367)$ and GluK4 $(\mathrm{p}=0.2785)$, compared to vehicle-treated controls (Fig. 4B). 
184 Amongst the 3 NMDA receptor subunits analysed treatment with paclitaxel significantly

185 increased the expression of GluN1 $(\mathrm{p}=0.0209)$ only, but not 2 subunits GluN2A $(\mathrm{p}=0.0612)$

186 and GluN2B ( $\mathrm{p}=0.1105)$, compared to vehicle-treated controls (Fig. 4C).

187 Of all the eight metabotropic glutamate receptors subunits quantified only $\mathrm{mGLuR}_{8}$ was

188 significantly altered $(\mathrm{p}=0.0144)$ in the ACC by treatment with paclitaxel compared to treatment

189 with vehicle (Figure 4E-F). Using the unpaired two-tailed Student's t-test the p values obtained

190 are: 0.4439 for $\mathrm{mGLuR}_{1}, 0.1340$ for $\mathrm{mGLuR}_{2}, 0.3201$ for $\mathrm{mGLuR}_{3}, 0.9971$ for $\mathrm{mGLuR}_{4}, 0.3375$

191 for $\mathrm{mGLuR}_{5}, 0.9693$ for $\mathrm{mGLuR}_{6}$ and 0.2780 for $\mathrm{mGLuR}_{7}$.

192 
193 Discussion

194 This is the first study to report on the quantification and/or changes in the transcript levels and

195 immunoreactivity of the astrocyte marker GFAP, transcript levels of glutamate transporters and

196 receptors in the ACC, an area associated with pain perception and modulation (Vogt 2005; Xie et

197 al. 2009; Zhuo 2008), during paclitaxel-induced neuropathic pain (PINP).

Increased expression of GFAP in the brain is a marker of astrocyte activation (Aldskogius \& Kozlova 1998). Various studies have reported increased expression of GFAP mRNA and protein in the ACC during pain (Chen et al. 2012; Kuzumaki et al. 2007; Lu et al. 2011). Astrocyte activation has also been observed in the ACC in other models of neuropathic pain (Xu et al. 2008; Yamashita et al. 2014) but had not been reported in PINP. However, astrocyte activation in the spinal cord has been reported to contribute to PINP in rodents (Ruiz-Medina et al. 2013; Zhang et al. 2012). In the current study the expression of GFAP transcripts and immunoreactivity in the ACC was increased in mice treated with PINP. During peripheral nerve injury neurons have been reported to release neurotransmitters such substance P and glutamate and neuronal chemokines that cause astrocyte activation in the CNS (Milligan \& Watkins 2009; Wang et al. 2009; Watkins et al. 2007). Activated astrocytes in turn release molecules that contribute to the pathophysiology of pain through modulation of neuronal functioning (Milligan \& Watkins 2009; Wang et al. 2009; Watkins et al. 2007). Thus, the current results suggest that astrocyte activation in the ACC might also contribute to the pathophysiology of PINP.

The activation of astrocytes in the spinal cord induced by paclitaxel has been reported to be accompanied with a decrease in the expression of the glial glutamate transporters GLAST and GLT-1 (Zhang et al. 2012) as well as an increase in the GABA transporter GAT-1 (Yadav et al. 
215 2015). In the current study, there were no changes in the transcript levels of glutamate

216 transporters in the ACC of paclitaxel-treated mice. However, in a recent study, we observed

217 elevated transcripts of GAT-1 in the ACC of mice with PINP (Masocha 2015). This suggests that

218 astrocyte activation and increased expression of GAT-1, but not glutamate transporters, in the

219 ACC play a role in the pathogenesis in PINP. This would result in an imbalance in the inhibitory

220 (GABA) and excitatory (glutamate) neurotransmitters, which might result in increased

221 excitability of the ACC. Increased neuronal excitability in the ACC might contribute to the

222 increased activity observed in the ACC during neuropathic pain in both humans and animal

223 models (Hsieh et al. 1995; Peyron et al. 2000; Tseng et al. 2013; Wrigley et al. 2009; Xu et al.

224 2008; Yamashita et al. 2014).

225 Although we did not observe any changes in the glutamate transporters in the ACC, we observed 226 that transcripts of various glutamate receptors and receptor subunits were elevated in the ACC of

227 mice with PINP. The increased expression of some of the glutamate receptors and receptor

228 subunits could have been linked to astrocyte activation since all of the up-regulated receptors are

229 expressed on astrocytes (Geurts et al. 2005; Martínez-Lozada \& Ortega 2015). Several receptors

230 have been reported to be differentially expressed in the ACC in rodent models of PINP. We

231 observed an increase in the expression of various GABA receptors in the ACC during PINP

232 (Masocha 2015). Ortega et al. reported a differential expression of muscarinic-1 and -2 receptors

233 and dopamine D1 and D2 receptors in the ACC of rodents with PINP (Ortega-Legaspi et al.

234 2011; Ortega-Legaspi et al. 2010). The increased expression glutamate receptors in the ACC also

235 suggest a role of the glutamatergic system in the pathogenesis of PINP. 


\section{Conclusions}

239 In conclusion, the results of this study show that animals with paclitaxel-induced neuropathic

240 pain (PINP) have increased transcripts and immunoreactivity of the astrocyte marker GFAP and

241 transcripts of some glutamate receptors and receptor subunits, but not glutamate transporters, in

242 the ACC. In a previous study, transcripts of a GABA transporter GAT-1, whose increase has

243 been associated with astrocyte activation in the spinal cord of rodents with PINP (Yadav et al.

244 2015), was found increased in the ACC of mice with PINP (Masocha 2015). Thus, inhibition of

245 astrocyte activation and GAT-1 activity and/or antagonism of specific glutamate receptors could

246 be therapeutic modalities of managing PINP and possibly other types on chemotherapy-induced

247 neuropathic pain.

248

249

250 


\section{Acknowledgements}

252 I am grateful to Dr Subramanian S Parvathy, Ms. Salini Soman from the Department of

253 Pharmacology and Therapeutics, Faculty of Pharmacy, and Ms. Jucy Gabriel from the Research

254 Core Facility, HSC, Kuwait University for their technical assistance and to the staff from the

255 Animal Resources Centre, HSC, Kuwait University for their support. 
257

258

259

260

261

262

263

264

265

266

267

268

269

270

271

272

273

274

275

276

277

278

279

280

281

282

283

284

285

286

287

288

289

290

291

292

293

294

295

296

297

298

299

Aldskogius H, and Kozlova EN. 1998. Central neuron-glial and glial-glial interactions following axon injury. Prog Neurobiol 55:1-26.

Chen FL, Dong YL, Zhang ZJ, Cao DL, Xu J, Hui J, Zhu L, and Gao YJ. 2012. Activation of astrocytes in the anterior cingulate cortex contributes to the affective component of pain in an inflammatory pain model. Brain Res Bull 87:60-66.

Collingridge GL, Olsen RW, Peters J, and Spedding M. 2009. A nomenclature for ligand-gated ion channels. Neuropharmacology 56:2-5.

Conn PJ, and Pin JP. 1997. Pharmacology and functions of metabotropic glutamate receptors. Annu Rev Pharmacol Toxicol 37:205-237.

Conti F, Melone M, De Biasi S, Minelli A, Brecha NC, and Ducati A. 1998. Neuronal and glial localization of GAT-1, a high-affinity gamma-aminobutyric acid plasma membrane transporter, in human cerebral cortex: with a note on its distribution in monkey cortex. J Comp Neurol 396:51-63.

Danbolt NC. 2001. Glutamate uptake. Prog Neurobiol 65:1-105.

Geurts JJ, Wolswijk G, Bo L, Redeker S, Ramkema M, Troost D, and Aronica E. 2005. Expression patterns of Group III metabotropic glutamate receptors mGluR4 and mGluR8 in multiple sclerosis lesions. J Neuroimmunol 158:182-190.

Gosselin RD, Bebber D, and Decosterd I. 2010. Upregulation of the GABA transporter GAT-1 in the gracile nucleus in the spared nerve injury model of neuropathic pain. Neurosci Lett 480:132-137.

Hsieh JC, Belfrage M, Stone-Elander S, Hansson P, and Ingvar M. 1995. Central representation of chronic ongoing neuropathic pain studied by positron emission tomography. Pain 63:225-236.

Kuzumaki N, Narita M, Hareyama N, Niikura K, Nagumo Y, Nozaki H, Amano T, and Suzuki T. 2007. Chronic pain-induced astrocyte activation in the cingulate cortex with no change in neural or glial differentiation from neural stem cells in mice. Neurosci Lett 415:22-27.

Livak KJ, and Schmittgen TD. 2001. Analysis of relative gene expression data using real-time quantitative PCR and the 2(-Delta Delta C(T)) Method. Methods 25:402-408.

Lu Y, Zhu L, and Gao YJ. 2011. Pain-related aversion induces astrocytic reaction and proinflammatory cytokine expression in the anterior cingulate cortex in rats. Brain Res Bull 84:178-182.

Maragakis NJ, and Rothstein JD. 2006. Mechanisms of Disease: astrocytes in neurodegenerative disease. Nat Clin Pract Neurol 2:679-689.

Martínez-Lozada Z, and Ortega A. 2015. Glutamatergic Transmission: A Matter of Three. Neural Plasticity 2015:787396.

Masocha W. 2009. Systemic lipopolysaccharide (LPS)-induced microglial activation results in different temporal reduction of CD200 and CD200 receptor gene expression in the brain. J Neuroimmunol 214:78-82.

Masocha W. 2014. Paclitaxel-induced hyposensitivity to nociceptive chemical stimulation in mice can be prevented by treatment with minocycline. Sci Rep 4:6719.

Masocha W. 2015. Comprehensive analysis of the GABAergic system gene expression profile in the anterior cingulate cortex of mice with Paclitaxel-induced neuropathic pain. Gene Expr 16:145153.

Meldrum BS. 2000. Glutamate as a neurotransmitter in the brain: review of physiology and pathology. $J$ Nutr 130:1007S-1015S.

Milligan ED, and Watkins LR. 2009. Pathological and protective roles of glia in chronic pain. Nat Rev Neurosci 10:23-36. 
300

301

302

303

304

305

306

307

308

309

310

311

312

313

314

315

316

317

318

319

320

321

322

323

324

325

326

327

328

329

330

331

332

333

334

335

336

337

338

339

340

341

342

343

344

345

346

347
Minelli A, Brecha NC, Karschin C, DeBiasi S, and Conti F. 1995. GAT-1, a high-affinity GABA plasma membrane transporter, is localized to neurons and astroglia in the cerebral cortex. J Neurosci 15:7734-7746.

Narita M, Kuzumaki N, Narita M, Kaneko C, Hareyama N, Miyatake M, Shindo K, Miyoshi K, Nakajima M, Nagumo Y, Sato F, Wachi H, Seyama Y, and Suzuki T. 2006. Chronic pain-induced emotional dysfunction is associated with astrogliosis due to cortical delta-opioid receptor dysfunction. $J$ Neurochem 97:1369-1378.

Nieto FR, Entrena JM, Cendan CM, Pozo ED, Vela JM, and Baeyens JM. 2008. Tetrodotoxin inhibits the development and expression of neuropathic pain induced by paclitaxel in mice. Pain 137:520531.

Niswender CM, and Conn PJ. 2010. Metabotropic glutamate receptors: physiology, pharmacology, and disease. Annu Rev Pharmacol Toxicol 50:295-322.

Ortega-Legaspi JM, de Gortari P, Garduno-Gutierrez R, Amaya MI, Leon-Olea M, Coffeen U, and Pellicer F. 2011. Expression of the dopaminergic D1 and D2 receptors in the anterior cingulate cortex in a model of neuropathic pain. Mol Pain 7:97.

Ortega-Legaspi JM, Leon-Olea M, de Gortari P, Amaya MI, Coffeen U, Simon-Arceo K, and Pellicer F. 2010. Expression of muscarinic $M 1$ and $M 2$ receptors in the anterior cingulate cortex associated with neuropathic pain. Eur J Pain 14:901-910.

Parvathy SS, and Masocha W. 2013. Matrix metalloproteinase inhibitor COL-3 prevents the development of paclitaxel-induced hyperalgesia in mice. Med Princ Pract 22:35-41.

Peters CM, Jimenez-Andrade JM, Kuskowski MA, Ghilardi JR, and Mantyh PW. 2007. An evolving cellular pathology occurs in dorsal root ganglia, peripheral nerve and spinal cord following intravenous administration of paclitaxel in the rat. Brain Res 1168:46-59.

Petroff OA. 2002. GABA and glutamate in the human brain. Neuroscientist 8:562-573.

Peyron R, Laurent B, and Garcia-Larrea L. 2000. Functional imaging of brain responses to pain. A review and meta-analysis (2000). Neurophysiol Clin 30:263-288.

Rothstein JD, Dykes-Hoberg M, Pardo CA, Bristol LA, Jin L, Kuncl RW, Kanai Y, Hediger MA, Wang Y, Schielke JP, and Welty DF. 1996. Knockout of glutamate transporters reveals a major role for astroglial transport in excitotoxicity and clearance of glutamate. Neuron 16:675-686.

Ruiz-Medina J, Baulies A, Bura SA, and Valverde O. 2013. Paclitaxel-induced neuropathic pain is age dependent and devolves on glial response. Eur J Pain 17:75-85.

Scripture CD, Figg WD, and Sparreboom A. 2006. Peripheral neuropathy induced by paclitaxel: recent insights and future perspectives. Curr Neuropharmacol 4:165-172.

Seifert G, Schilling K, and Steinhauser C. 2006. Astrocyte dysfunction in neurological disorders: a molecular perspective. Nat Rev Neurosci 7:194-206.

Senapati AK, Lagraize SC, Huntington PJ, Wilson HD, Fuchs PN, and Peng YB. 2005. Electrical stimulation of the anterior cingulate cortex reduces responses of rat dorsal horn neurons to mechanical stimuli. J Neurophysiol 94:845-851.

Sewards TV, and Sewards MA. 2002. The medial pain system: neural representations of the motivational aspect of pain. Brain Res Bull 59:163-180.

Shigeri Y, Seal RP, and Shimamoto K. 2004. Molecular pharmacology of glutamate transporters, EAATs and VGLUTs. Brain Res Brain Res Rev 45:250-265.

Tseng MT, Chiang MC, Chao CC, Tseng WY, and Hsieh ST. 2013. fMRI evidence of degeneration-induced neuropathic pain in diabetes: Enhanced limbic and striatal activations. Hum Brain Mapp 34:2733-2746.

Vogt BA. 2005. Pain and emotion interactions in subregions of the cingulate gyrus. Nat Rev Neurosci 6:533-544.

Wang DD, and Bordey A. 2008. The astrocyte odyssey. Prog Neurobiol 86:342-367. 
Wang W, Wang W, Mei X, Huang J, Wei Y, Wang Y, Wu S, and Li Y. 2009. Crosstalk between spinal astrocytes and neurons in nerve injury-induced neuropathic pain. PLoS One 4:e6973.

Watkins LR, Hutchinson MR, Ledeboer A, Wieseler-Frank J, Milligan ED, and Maier SF. 2007. Norman Cousins Lecture. Glia as the "bad guys": implications for improving clinical pain control and the clinical utility of opioids. Brain Behav Immun 21:131-146.

Weng HR, Aravindan N, Cata JP, Chen JH, Shaw AD, and Dougherty PM. 2005. Spinal glial glutamate transporters downregulate in rats with taxol-induced hyperalgesia. Neurosci Lett 386:18-22.

Wolf S, Barton D, Kottschade L, Grothey A, and Loprinzi C. 2008. Chemotherapy-induced peripheral neuropathy: prevention and treatment strategies. Eur J Cancer 44:1507-1515.

Wrigley PJ, Press SR, Gustin SM, Macefield VG, Gandevia SC, Cousins MJ, Middleton JW, Henderson LA, and Siddall PJ. 2009. Neuropathic pain and primary somatosensory cortex reorganization following spinal cord injury. Pain 141:52-59.

Xie YF, Huo FQ, and Tang JS. 2009. Cerebral cortex modulation of pain. Acta Pharmacol Sin 30:31-41.

Xu H, Wu L, Wang H, Zhang X, Vadakkan KI, Kim SS, Steenland HW, and Zhuo M. 2008. Presynaptic and postsynaptic amplifications of neuropathic pain in the anterior cingulate cortex. $J$ Neurosci 28:7445-7453.

Yadav R, Yan X, Maixner DW, Gao M, and Weng HR. 2015. Blocking the GABA transporter GAT-1 ameliorates spinal GABAergic disinhibition and neuropathic pain induced by paclitaxel. $J$ Neurochem 133:857-869.

Yamashita A, Hamada A, Suhara Y, Kawabe R, Yanase M, Kuzumaki N, Narita M, Matsui R, Okano H, and Narita M. 2014. Astrocytic activation in the anterior cingulate cortex is critical for sleep disorder under neuropathic pain. Synapse 68:235-247.

Yi JH, Pow DV, and Hazell AS. 2005. Early loss of the glutamate transporter splice-variant GLT-1v in rat cerebral cortex following lateral fluid-percussion injury. Glia 49:121-133.

Zhang H, Yoon SY, and Dougherty PM. 2012. Evidence that spinal astrocytes but not microglia contribute to the pathogenesis of Paclitaxel-induced painful neuropathy. J Pain 13:293-303.

Zhuo M. 2008. Cortical excitation and chronic pain. Trends Neurosci 31:199-207.

Zimmermann M. 1983. Ethical guidelines for investigations of experimental pain in conscious animals. Pain 16:109-110. 


\section{Figure 1 (on next page)}

Effects of paclitaxel on glial fibrillary acidic protein (GFAP) transcript levels in the anterior cingulate cortex (ACC)

Relative GFAP mRNA expression in the ACC of BALB/C mice on day 7 after first administration of the drug or its vehicle. Each point represents the mean \pm S.E.M of the values obtained from 21 vehicle-treated control mice and 24 paclitaxel-treated mice. $* * p<0.01$ compared to vehicle-treated control mice. 

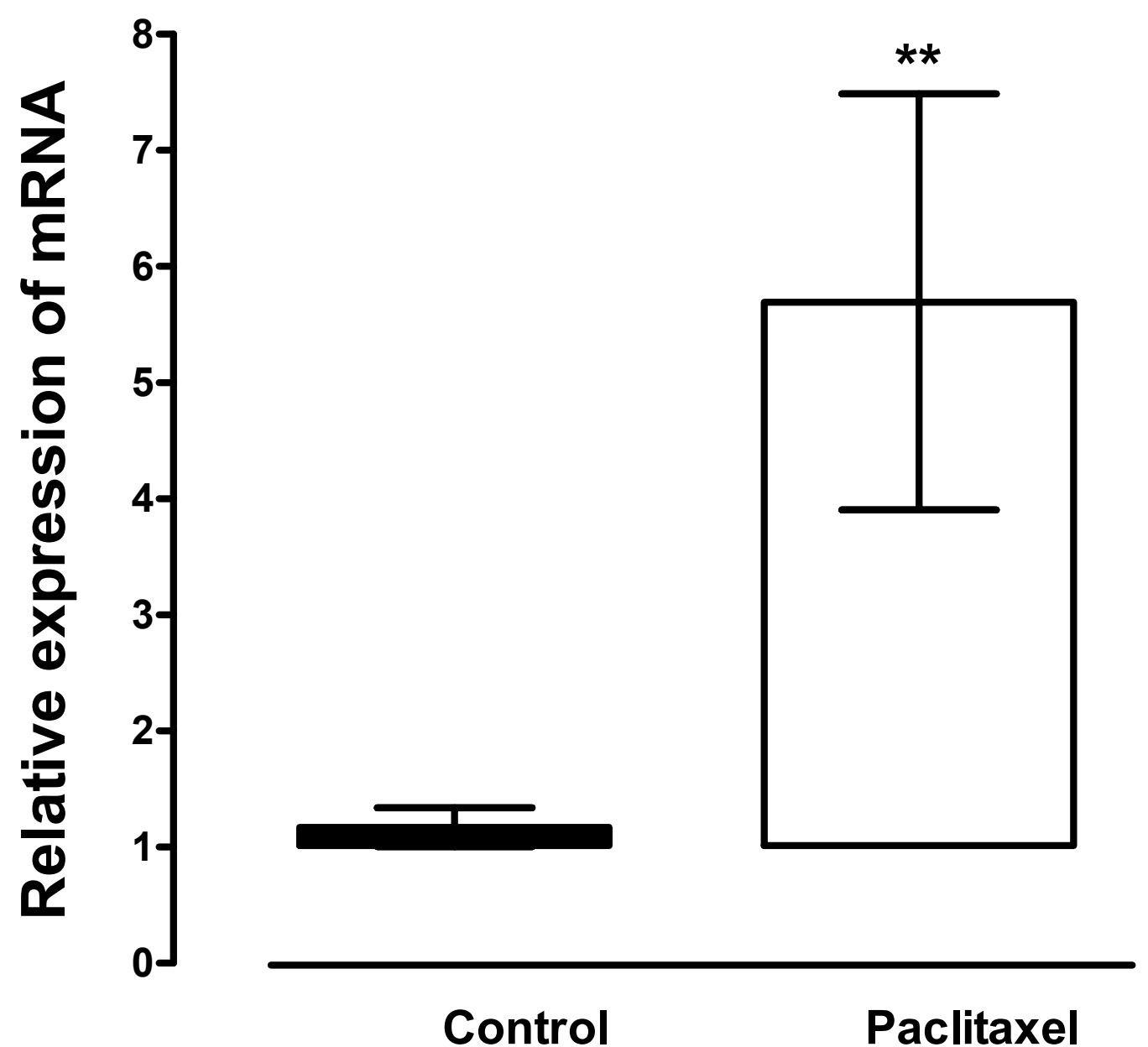
2

Effects of paclitaxel on glial fibrillary acidic protein (GFAP) immunoreactivity in the anterior cingulate cortex (ACC)

GFAP immunoreactivity in the ACC of BALB/C mice on day 7 after first administration of the drug or its vehicle. GFAP immunoreactivity in astrocytes is increased in 3 paclitaxel-treated mice (D -F) compared to 3 vehicle-treated control mice $(A-C)$ in the ACC. Note that in a paclitaxel-treated mouse (D) increased immunoreactivity of GFAP appears to be along a blood vessel: Scale bar: $50 \mu \mathrm{m}$.
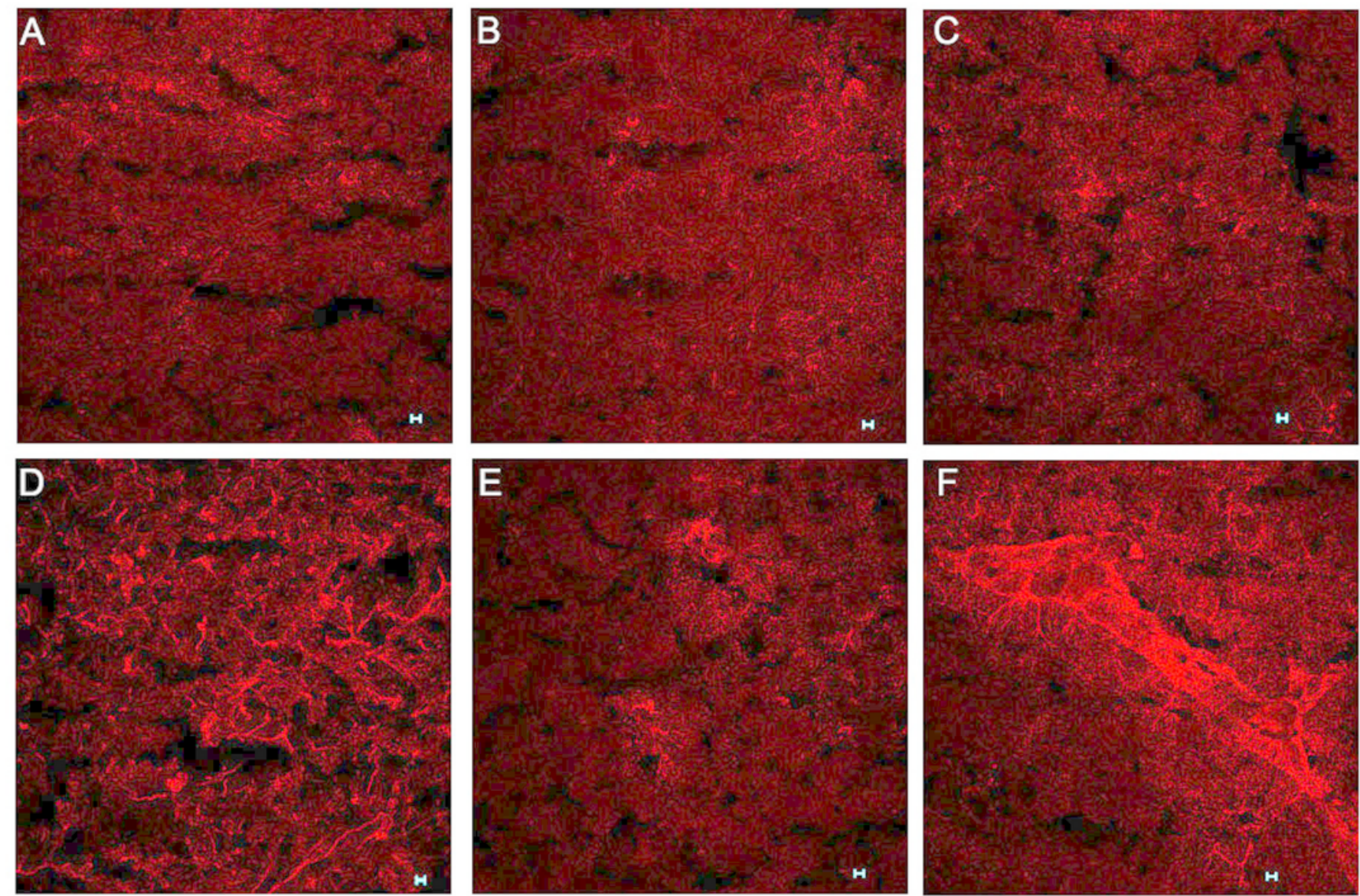


\section{Figure 3 (on next page)}

Effects of paclitaxel on glutamate transporters transcript levels in the anterior cingulate cortex (ACC)

Relative mRNA expression of (A) excitatory amino acid transporters GLAST, GLT-1, EAAC1, EAAT4, and (B) vesicular glutamate transporters VGLUT1 and VGLUT2 in the ACC of BALB/C mice on day 7 after first administration of the drug or its vehicle. Each point represents the mean \pm S.E.M of the values obtained from 11-15 vehicle-treated control mice and 13-15 paclitaxel-treated mice . 


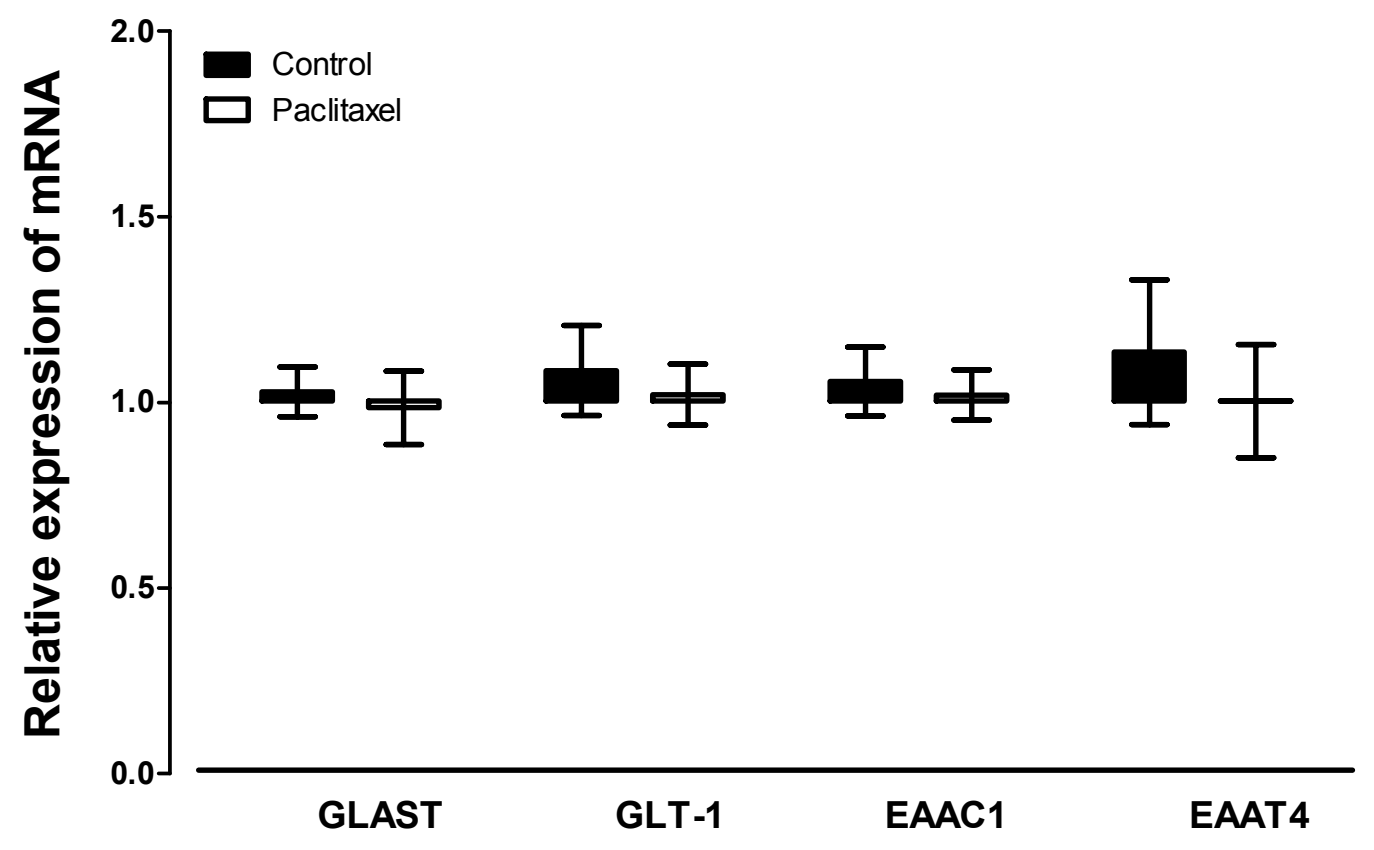

B

VGLUTS

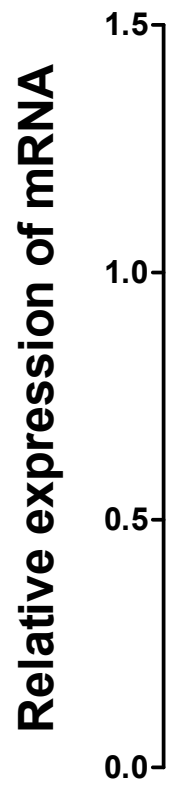

1.57

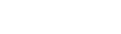


Figure 4 (on next page)

Effects of paclitaxel on glutamate receptors transcript levels in the anterior cingulate cortex (ACC)

Relative mRNA expression of (A) AMPA receptor subunits GLuA1 to 3, (B) kainate receptor subunits GLuK1 to 5, (C) NMDA receptor subunits GLuN1, GLuN2A and GLuN2B, and (D-F) metabotropic glutamate receptors $\mathrm{mGLuR}_{1}$ to ${ }_{8}$ in the ACC of BALB/c mice on day 7 after first administration of the drug or its vehicle. Each point represents the mean \pm S.E.M of the values obtained from 6-15 vehicle-treated control mice and 8-16 paclitaxel-treated mice. $* p<0.05, * * p<0.01$ compared to vehicle-treated control mice. 


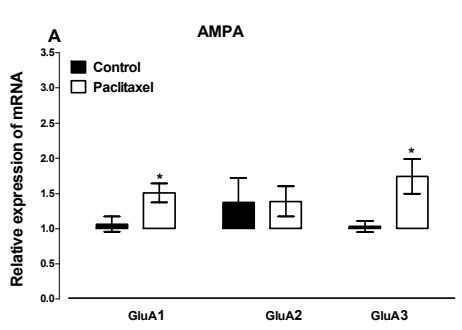

PeerJ Kainate Manuscript to be reviewed
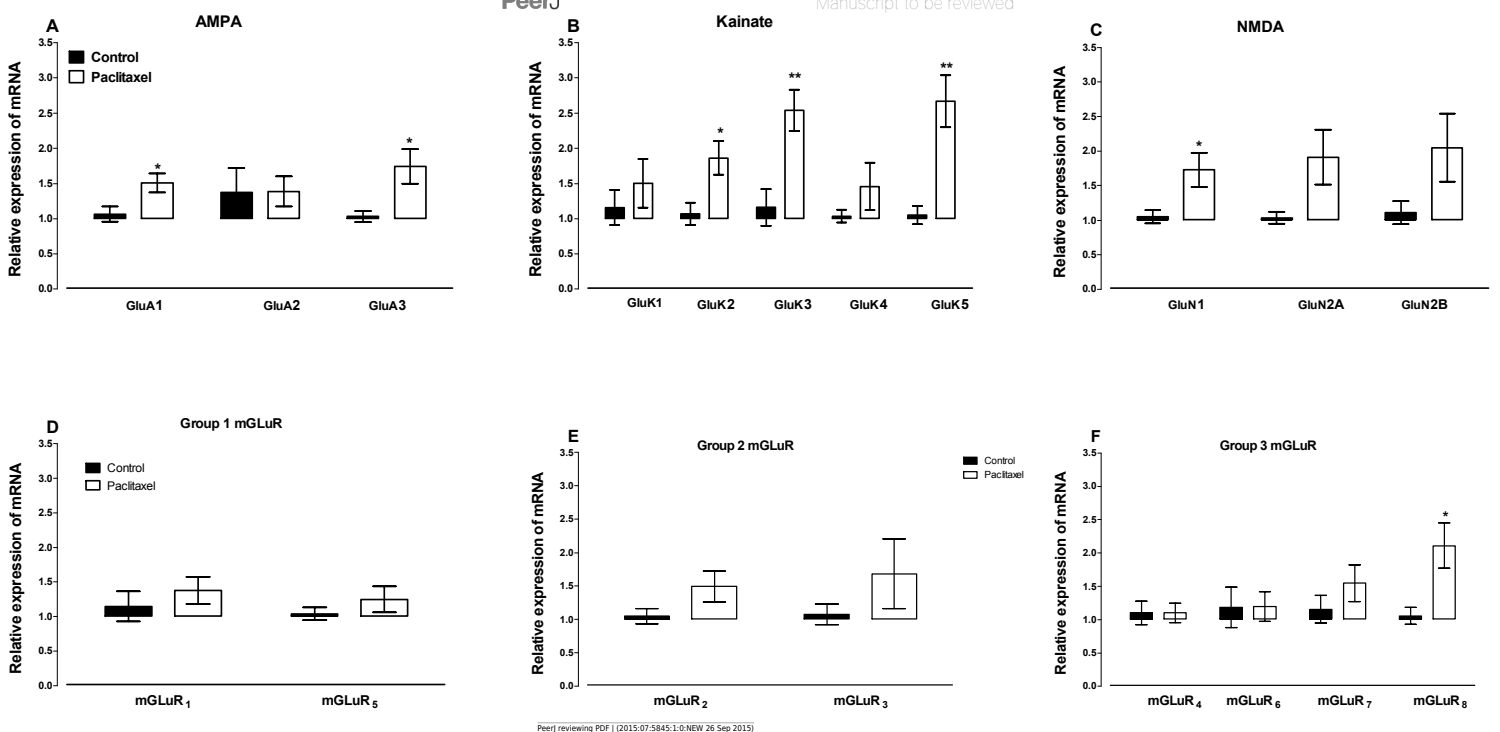


\section{Table $\mathbf{1}$ (on next page)}

PCR primer sequences of cyclophilin, GFAP and glutamatergic system molecules 
1 Table 1 PCR primer sequences of cyclophilin, GFAP and glutamatergic system molecules

\begin{tabular}{|l|l|l|}
\hline \multirow{2}{*}{ Gene } & Polarity & \multicolumn{2}{l|}{} \\
\cline { 2 - 3 } & $\begin{array}{l}\text { Sense } \\
\text { Sequence } \mathbf{5}^{\prime} \text { to } \mathbf{3}^{\prime}\end{array}$ & $\begin{array}{l}\text { Anti-sense } \\
\text { sequence } \mathbf{5}^{\prime} \text { to } \mathbf{3}^{\prime}\end{array}$ \\
\hline Cyclophilin & GCTTTTCGCCGCTTGCT & CTCGTCATCGGCCGTGAT \\
\hline GFAP & ACAGCGGCCCTGAGAGAGAT & CTCCTCTGTCTCTTGCATGTTACTG \\
\hline GLAST & ACCAAAAGCAACGGAGAAGAG & GGCATTCCGAAACAGGTAACTC \\
\hline GLT-1 & ACAATATGCCCAAGCAGGTAGA & CTTTGGCTCATCGGAGCTGA \\
\hline EAAC1 & CTTCCTACGGAATCACTGGCT & CGATCAGCGGCAAAATGACC \\
\hline EAAT4 & AGCAGCCACGGCAATAGTC & ATGCCAAGCTGACACCAATGA \\
\hline VGLUT-1 & GGTGGAGGGGGTCACATAC & AGATCCCGAAGCTGCCATAGA \\
\hline VGLUT-2 & CCCTGGAGGTGCCTGAGAA & GCGGTGGATAGTGCTGTTGTT \\
\hline GLuA1 & CCGTTGACACATCCAATCAGTTT & GTCGATAATGCTAATGAGAGCTTCCT \\
\hline GLuA2 & AAATTGCCAAACATTGTGG & ATGGAGCCATGGCAATATCA \\
\hline GLuA3 & ACACCATCAGCATAGGTGGA & TCAGTGGTGTTCTGGTTGGT \\
\hline GLuA4 & TTGGAATGGGATGGTAGGAG & TAGGAACAAGACCACGCTGA \\
\hline GLuK1 & TCACACCCTACGAGTGGTATAAC & AGCTCCAACGCCAAACCAG \\
\hline GLuK2 & ATCGGATATTCGCAAGGAACC & CCATAGGGCCAGATTCCACA \\
\hline GLuK3 & AGGTCCTAATGTCACTGACTCTC & GCCATAAAGGGTCCTATCAGAC \\
\hline GLuK4 & CCAAGGTCGAAGTGGACATCT & CTGGGGTGAAGGTTCAGGG \\
\hline GLuK5 & ATAGTCGCCTTCGCCAATCC & GTGTCCGTGGTCTCGTACTG \\
\hline GLuN1 & GGCATCGTAGCTGGGATCTTC & TCCTACGGGCATCCTTGTG \\
\hline GLuN2A & GTTTGTGGTGACGGTGAGA & AAGAGGTGCTCCCAGATGAA \\
\hline GLuN2B & ATGTGGATTGGGAGGATAGG & TCGGGCTTTGAGGATACTTG \\
\hline mGluR1 & TGTCATCAACGCCATCTATGC & CCCACGTAGCCAGGACATAGAG \\
\hline mGluR2 & CGCTCTCTGCACGCTCTATG & GATGAACTTGGCCTCGTTGAA \\
\hline mGluR3 & AAGCCATCGCCTGTCATCTG & GGAGGTCCCAAGCCCAAGT \\
\hline mGluR4 & GATGCTCTACATGCCCAAAGTCTAC & CGGTGACAACGGCTTTGAG \\
\hline mGluR5 & TGACCCTGAGCCCATTGC & AACGAAGAGGGTGGCTAGCA \\
\hline mGluR6 & TCATGGCCACCACAACTATCA & CAGAGGCGCGGACTATGG \\
\hline mGluR7 & AAGCCTGGGCAGAGGAAGA & TCCATCACAGGGCTCACAAG \\
\hline mGluR8 & CAGCATCTGTCTGCAGCCTG & CGGTTTTCTTCCTCTCCCCA \\
\hline & & \\
\hline
\end{tabular}

